\title{
NY-ESO-1b Peptide Vaccine
}

National Cancer Institute

\section{Source}

National Cancer Institute. NY-ESO-1b Peptide Vaccine. NCI Thesaurus. Code C68999.

A recombinant nonapeptide used as an antineoplastic vaccine. NY-ESO-1 b peptide vaccine contains the amino acid sequence SLLMWIT QC, derived from the cancer-testis tumor antigen (NY-ESO-1), which is expressed on tumor cells of many different types, including melanomas. Vaccination with this peptide vaccine may elicit strong humoral and cellular immune responses to NY-ESO-1-expressing cancers. 